\title{
GENETICS
}

\section{ALK takes the rap}

Like retinoblastoma, neuroblastoma can be an inherited or a sporadic tumour but, unlike retinoblastoma, the genetics underlying neuroblastoma development have remained for the most part enigmatic. Four papers published in Nature have now found at least one major culprit - the anaplastic lymphoma kinase (ALK).

Using a variety of genome-wide screens, the groups variously found that $A L K$ is mutated in $6-12 \%$ of sporadic cases, and the analyses by John Maris and colleagues showed that $A L K$ is a major familial neuroblastoma predisposition gene. ALK is either amplified or constitutively activated through mutations mainly within the kinase domain, phenylalanine 1174 and arginine 1275 being two mutation hotspots. Expression of $A L K$ mutants in a variety of cell lines resulted in cellular transformation and tumour formation in vivo. RNA inhibition of $A L K$ in these cells and in neuroblastoma cell lines with $A L K$ mutations resulted predominantly

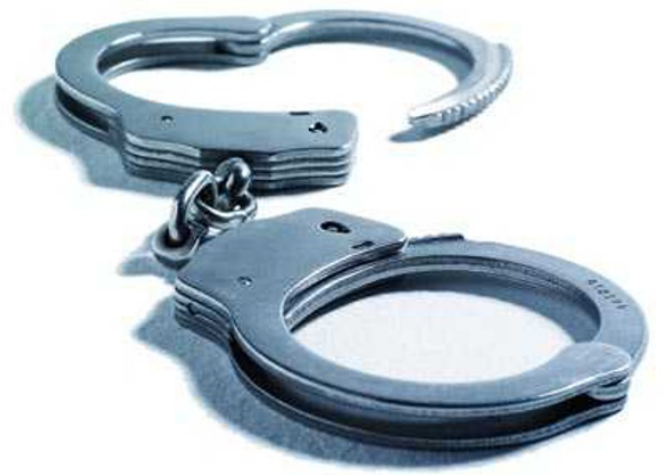

PHOTOALTO in growth arrest, with apoptosis being evident in a few cases. Because ALK is activated by chromosomal translocations in a number of other tumour types, small molecular targeted drugs are being developed to target this kinase. The use of one of these, TAE684, resulted in growth suppression and cell death.

Although neuroblastoma shows a wide range of clinical phenotypes, including a few cases that spontaneously regress, $40-50 \%$ of children present with aggressive, metastatic disease, often associated with amplification of $M Y C N$. Mutation and amplification of $A L K$ is also associated with the more aggressive subtypes of this disease. However, whether ALK mutation is an independent risk factor for poor prognosis, like $M Y C N$, is yet to be determined. Hopefully these findings will encourage further work in the development of ALK-targeted drugs, with a view to improving the outcome for children with more aggressive stages of neuroblastoma.

Nicola McCarthy

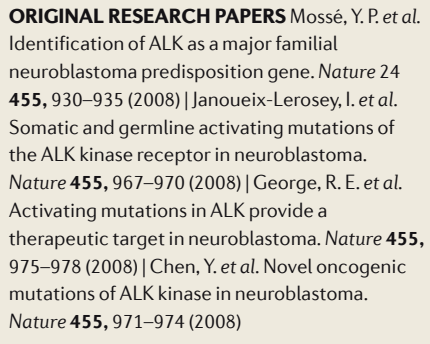

